\title{
Gel-like inclusions of C-terminal fragments of TDP-43 sequester and inhibit proteasomes in neurons
}

Henrick Riemenschneider ${ }^{1, *}$, Qiang Guo ${ }^{2,3, *}$, Jakob Bader ${ }^{4}$, Frédéric Frottin ${ }^{5,6}$, Daniel Farny ${ }^{1}$, Gernot Kleinberger ${ }^{1}$, Christian Haass ${ }^{1,7,8}$, Matthias Mann ${ }^{4}$, F. Ulrich Hartt ${ }^{5,8}$, Wolfgang Baumeister ${ }^{2}$, M ark S. Hipp $^{5,9,10}$, Felix M eissner ${ }^{4}$, Ruben Fernandez-Busnadiego 2,11,12,\#, Dieter Edbauer 1,8,13,\#

1 German Center for Neurodegenerative Diseases (DZNE), M unich, Feodor-Lynen-Str. 17, 81377

Munich, Germany

2 Department of M olecular Structural Biology, M ax Planck Institute of Biochemistry, 82152

Martinsried, Germany

3 State Key Laboratory of Protein and Plant Gene Research, School of Life Sciences and Peking-

Tsinghua Center for Life Sciences, Peking University, Beijing 100871, China

4 Department of Proteomics and Signal Transduction, M ax Planck Institute for Biochemistry, Am

Klopferspitz 18, 82152 M artinsried, Germany

5 Department of Cellular Biochemistry, M ax Planck Institute for Biochemistry, Am Klopferspitz 18,

82152 Martinsried, Germany

6 Université Paris-Saclay, CEA, CNRS, Institute for Integrative Biology of the Cell (I2BC), 91198, Gifsur-Yvette, France.

7 Chair of M etabolic Biochemistry, Biomedical Center (BMC), Faculty of M edicine, Ludwig-

Maximilians-Universität M unich, Germany

8 M unich Cluster of Systems Neurology (SyNergy), Feodor-Lynen-Str. 17, 81377 M unich, Germany

9 Department of Biomedical Sciences of Cells and Systems, University M edical Center Groningen,

University of Groningen, Antonius Deusinglaan 1, 9713 AV Groningen, The Netherlands

10 School of M edicine and Health Sciences, Carl von Ossietzky University Oldenburg, Oldenburg,

Germany

11 Institute of Neuropathology, University M edical Center Göttingen, 37099 Göttingen, Germany

12 Cluster of Excellence "M ultiscale Bioimaging: from M olecular Machines to Networks of Excitable

Cells" (M BExC), University of Göttingen, 37075 Göttingen, Germany

13 Ludwig-M aximilians-University M unich, Graduate School of Systemic Neurosciences (GSN), 81377

Munich, Germany

* These authors contributed equally.

\#Correspondence should be addressed to dieter.edbauer@dzne.de and

ruben.fernandezbusnadiego@med.uni-goettingen.de 


\section{Abstract}

TDP-43 inclusions enriched in C-terminal fragments of 25kDa ("TDP-25") are associated with neurodegeneration in amyotrophic lateral sclerosis (ALS) and frontotemporal dementia (FTD). Here, we analyzed gain-of-function mechanisms of TDP-25 combining cryo-electron tomography, proteomics and functional assays. TDP-25 inclusions are amorphous with gel-like biophysical properties and sequester proteasomes adopting exclusively substrate-processing conformations. This leads to proteostasis impairment, further enhanced by pathogenic mutations. These findings bolster the importance of proteasome dysfunction in ALS/FTD.

\section{Introduction}

TDP-43 aggregation is the disease-defining pathological hallmark in $90 \%$ of patients with amyotrophic lateral sclerosis (ALS) and $\sim 45 \%$ of patients with frontotemporal dementia (FTD) (Gao et al, 2017; Prasad et al, 2019). However, we still have a limited understanding of the native structure of TDP-43 inclusions and their role in disease (Gao et al, 2017). Predominantly cytoplasmic neuronal inclusions of TDP-43 correlate strongly with regional neuron loss in spinal cord, motor cortex or frontal-temporal cortical regions (M ackenzie et al, 2013). These inclusions are enriched in 25-35 kDa C-terminal fragments of TDP-43 that contain a glycine-rich low-complexity region (Igaz et al, 2008). Rare autosomal dominant ALS-causing mutations in TDP-43 cluster in this region, although their pathomechanism is still poorly understood, and they have only modest effects in vitro and in knockin models (Prasad et al, 2019). However, overexpression of the inclusion-forming 25kDa Cterminal fragment ("TDP-25") triggers neurodegeneration in mice even without disease-associated mutations (Walker et al, 2015).

Distinct biophysical mechanisms can drive inclusion formation in the context of neurodegenerative diseases: (i) formation of highly insoluble amyloid fibrils with cross $\beta$-sheet conformation (Eisenberg \& Jucker, 2012), (ii) liquid-liquid phase separation (LLPS) into highly dynamic liquid-like droplets (Gomes \& Shorter, 2019), which may solidify and adopt amyloid-like conformations under pathological conditions (Kato et al, 2012; Patel et al, 2015; Qamar et al, 2018). In vitro, full length TDP-43 can form liquid droplet through LLPS but short peptide fragments from the C-terminal region form amyloids (Cao et al, 2019; Conicella et al, 2016). By adopting aberrant conformations, aggregated proteins may engage in toxic cellular interactions (Hipp et al, 2019; Olzscha et al, 2011). Pathogenic mutations in rare familial cases of ALS/FTD are linked to the ubiquitin-proteasome system (UPS) and autophagy, which can clear aggregated and phase-separated proteins, suggesting 
the proteostasis system is of particular importance at least in genetic ALS/FTD (Deng et al, 2011; Gitcho et al, 2009; Hipp et al, 2019). We have previously shown that poly-GA inclusions in C9orf72 ALS/FTD disrupt neuronal proteostasis by sequestering proteasomes in a rare transition state, suggesting the cyclic conformational changes required for substrate processing (Collins \& Goldberg, 2017) are blocked by the inclusions (Guo et al, 2018).

\section{TDP-25 forms amorphous inclusions enriched in proteasomes}

Here, we aimed to elucidate gain-of-function mechanisms as well as the structure of cytoplasmic TDP-43 aggregates found in sporadic and genetic ALS/FTD focusing on the aggregation-prone TDP-25 fragment (residues 220-414 of full length human TDP-43) (Zhang et al, 2009). Expression of GFPtagged TDP-25 in rat primary neurons resulted in abundant cytoplasmic inclusions phosphorylated at disease-specific sites (Fig. 1A) (Hasegawa et al, 2008). To determine which pathomechanisms are enhanced by known pathogenic mutations, we additionally used a TDP-25 variant containing eight mutations (G290A, G294V, G298S, A315T, M 337V, G348C, N352S, A382T) that individually cause ALS (Prasad et al, 2019). Wild-type and mutant GFP-TDP-25 formed inclusions of similar appearance by light microscopy (Fig. 1A). While GFP-TDP-43 was almost completely soluble in RIPA buffer, a large fraction of wild-type and mutant GFP-TDP-25 was only solubilized upon sequential extraction of the RIPA-insoluble material with 2\% SDS (Fig. 1B) indicative of stronger intermolecular interactions in aggregating TDP-25.

To investigate the downstream consequences of TDP-25 aggregation, we analyzed neuronal GFPTDP-25 inclusions in situ using cryo-electron tomography (cryo-ET), a powerful method to visualize aggregates within their native cellular environment (Bauerlein et al, 2017; Guo et al, 2018). TDP-25 inclusions appeared amorphous and seemingly lacked fibrillar structure, although they were clearly demarcated within the cytoplasm (Fig. 1C/D, M ovie S1). These findings are in contrast to poly-Q, poly-GA and $\alpha$-synuclein neuronal inclusions, which show amyloid-like conformation in neurons in our cryo-ET pipeline (Bauerlein et al, 2017; Guo et al, 2018; Trinkaus et al, 2020). Introducing the eight ALS-causing mutations had no obvious effect on the ultrastructure of TDP-25 inclusion (Fig. S1A).

Similar to our findings in C9orf72 poly-GA inclusions (Guo et al, 2018), we detected ring-like structures (Fig. 1C) accumulating within TDP-25 inclusions. Sub-tomogram averaging of these rings (Fig. S1B) converged to a proteasome structure at $\sim 20 \AA$ resolution (Fig. S1C). Interestingly, an extra density that was not accounted for by proteasomal subunits was present on the proteasome regulatory particle, possibly reflecting substrates or adaptor proteins (Fig. 1E). Whereas ribosomes were largely excluded from TDP-25 inclusions, we found a $\sim 8$-fold enrichment of proteasomes 
compared to proteasome concentration in control neurons (Asano et al, 2015) (Fig. 1D and S1D).

Strikingly, virtually all proteasome particles within the inclusions were in substrate processing states, based on the conformation of 195 regulatory particles (Fig. 1E/F). In comparison, only $20 \%$ and $37 \%$ of proteasomes were in substrate-processing states in control neurons and poly-GA inclusions, respectively (Asano et al, 2015; Guo et al, 2018) (Fig. 1F). The lack of detectable ground state proteasomes suggests that proteasomes inside TDP-25 inclusions are stalled, as proteasome function requires cyclic transition through activated and ground states (Collins \& Goldberg, 2017).

\section{TDP-25 inclusions are gel-like}

Given the amorphous appearance of TDP-25 inclusions, we asked whether they may represent phase-separated liquid droplets. Thus, we analyzed the mobility of GFP-TDP-25 in neuronal inclusions using fluorescence recovery after photobleaching (FRAP) in comparison with known liquid and solid reference proteins, i.e. nucleolar NPM 1 and inclusion-forming poly-Q, respectively (Bauerlein et al, 2017; Frottin et al, 2019). In contrast to TDP-25, GFP-NPM 1 fluorescence recovered within seconds after bleaching, consistent with high mobility of the protein within the liquid-like nucleolus (Fig. 2A/B). This clearly argues against a liquid droplet character of TDP-25 inclusions. In contrast, nuclear full-length TDP-43 showed a much higher mobile fraction (Fig. S2), in line with its good solubility (Fig. 1B). However, compared to fibrillar poly-Q (huntingtin exon 1 containing 97 glutamines fused to GFP; Htt97Q-GFP), TDP-25 mobility was much higher ( $\sim 10 \%$ vs $\sim 25 \%$ recovery at $22 \mathrm{~min}$, Fig. 2C/D). These findings suggest that non-fibrillar TDP-25 adopts a conformation that is best described by a gel-like state. Solidification into hydrogels has been reported for phase separated droplets of FUS, but involves formation of amyloid-like fibrils (M urray et al, 2017; Qamar et al, 2018). A similar process has been postulated for TDP-43, although fibril formation was only detected for much shorter peptides in vitro (Cao et al, 2019; Gasset-Rosa et al, 2019; Guenther et al, 2018; Mann et al, 2019). Taken together, GFP-TDP-25 inclusions are clearly less dynamic than classical phase separated compartments, but more dynamic than fibrillar aggregates.

\section{TDP-25 sequesters and inhibits the proteasome}

Phase-separated inclusions may form through weak multivalent interactions with other cellular components (Gomes \& Shorter, 2019; Martin \& Mittag, 2018). To further investigate this gel-like state and the molecular consequences of the TDP-25 inclusions, we analyzed the interactome of TDP-25 and full length TDP-43 using mass-spectrometry-based proteomics. We detected several hundred specific interactors for full length TDP-43 and TDP-25 in primary neurons, in comparison to the GFP-only control (Fig. 3A/B and S3A, Table S1). TDP-43 specific interactors were strongly 
enriched in splicing-associated proteins consistent with the prominent involvement of TDP-43 in splicing (Fig. 3A/D) (Prasad et al, 2019).

The loss of the N-terminus in TDP-25 led to the loss of 72 interactors compared to full-length TDP-43 but resulted in over 400 new interactions, which may shape biophysical properties and drive gain-offunction toxicity in ALS/FTD (Fig. 3B). To investigate the role of LLPS in inclusion formation in our neuronal model, we analyzed the content of low-complexity domains or intrinsically disordered regions in interacting proteins, as such sequences drive phase separation through multivalent homoor heterotypic interactions (Martin \& Mittag, 2018). Interestingly, the proportion of disordered regions/low-complexity regions was lower in TDP-25 specific interactors than in TDP-43 specific and shared interactors (Fig. 3C), consistent with the higher dynamics of nuclear TDP-43 (Fig. S2). The relative absence of proteins with low-complexity domains within TDP-25 inclusions argues against their formation through LLPS, or may reflect solidification driven by loss of LLPS-promoting lowcomplexity interactors.

Consistent with our cryo-ET data, proteomics showed that proteasome subunits were highly enriched in the TDP-25 interactome (Fig. 3A/D and Table S2). Furthermore, immunofluorescence confirmed partial sequestration of proteasomes into GFP-TDP-25 inclusions (Fig. S3B), similar to poly-GA inclusions in C9orf72 ALS/FTD (Guo et al, 2018). Our mass spectrometry data also confirmed the previously reported selective interaction of TDP-25 with other UPS components, such as the ALSlinked Ubiquilin 2 (Cassel \& Reitz, 2013; Deng et al, 2011) and the E3 ubiquitin ligases Pja1 (Watabe et al, 2020). These or other UBL domain proteins may contribute to the extra density found on the proteasome regulatory particle (Fig. 1E).

Finally, we investigated the functional consequences of proteasome recruitment to TDP-25 inclusions and asked whether TDP-25 is primarily a substrate or an inhibitor of the proteasome. Treating GFP-TDP-25 expressing neurons with the proteasome inhibitor M G132 increased the level of punctate GFP-TDP-25, without obvious effects on the larger inclusions (Fig. 3E), in line with a previous report (Scotter et al, 2014). Quantification showed a similar 2-fold accumulation of GFPTDP-25 wild-type and mutant upon proteasome inhibition, suggesting that the proteasome is actively degrading largely soluble GFP-TDP-25 under basal conditions.

Our cryo-ET data suggested alterations in the functional cycle of proteasomes recruited to TDP-25 inclusions. To interrogate whether TDP-25 affects the cellular proteasome capacity, we expressed RFP-TDP-25 in an established UbG76V-GFP reporter HEK293 cell line (Dantuma et al, 2000). We noticed a dose-dependent accumulation of the UPS reporter in cells expressing RFP-TDP-25 wild- 
type and mutant, indicative of competitive inhibition (Fig. 3F). At high expression levels, mutant TDP25 impaired overall protein degradation significantly more than wild-type, arguing for a potentially disease-relevant mechanism. These findings corroborate our structural data, indicating that proteasomes enriched in TDP-25 inclusions are functionally impaired.

\section{Conclusion}

Using cryo-ET, proteomics and functional assays in primary neurons, we show that TDP-43 Cterminal fragments adopt a gel-like conformation and disrupt proteostasis by sequestering stalled proteasomes. This gel-like conformation may either reflect a transition state from a liquid compartment to mature aggregates or an alternative pathway to aggregation similar to the physiological cytoplasmic myo-granules of full-length TDP-43 found in regenerating muscle, which are SDS-resistant but lack classical amyloid conformation (Vogler et al, 2018). None of the cytoplasmic inclusions of TDP-25 showed the distinct anisosome-like structure containing a TDP-43 shell and a HSP70 core recently described for RNA-free TDP-43 in the nucleus (Yu et al, 2021), although both TDP-25 and TDP-43 interacted with HSP70 proteins in our model (Hspala and Hspa8, see Fig. 3A). Thus, the disease-associated proteolytic fragmentation and cytoplasmic localization of TDP-43 fundamentally change its biophysical properties. Interestingly, so far cryo-ET has only detected proteasome accumulation and impairment within inclusions related to ALS/FTD ((Guo et al, 2018) and this manuscript), but not in other protein aggregates such as poly-Q or $\alpha$-synuclein (Bauerlein et al, 2017; Schaefer et al, 2019; Trinkaus et al, 2020). Additionally, we show that ALScausing mutations may further impair proteasome function. These data resonate with previous reports on the crucial role of the UPS for motor neuron function and survival (Bax et al, 2019; Bett et al, 2009; Tashiro et al, 2012), pointing to proteasome dysfunction as a major specific hallmark of ALS/FTD pathogenesis. 


\section{Acknowledgements}

We thank Philipp Erdmann, Günter Pfeifer, Jürgen Plitzko and M iroslava Schaffer for electron microscopy support. We thank the Imaging Facility of the M ax Planck Institute of Biochemistry. We thank Christian Behrends, M areike Czuppa, Dorothee Dormann, Bettina Schmid, Ali Rezaei and Qihui Zhou for critical comments to the manuscript. This work was supported by NOM IS foundation (D.E.). M.S.H., F.U.H., Q.G., W.B. and R.F.-B. have received funding from the European Commission (FP7 GA ERC-2012-SyG_318987-ToPAG). D.E., C.H., M.S.H., F.U.H. and R.F.-B. acknowledge funding from the Deutsche Forschungsgemeinschaft (DFG, German Research Foundation) through Germany's Excellence Strategy - EXC 2067/ 1- 390729940 (R.F.-B.) and EXC 2145 - 390857198 (C.H., F.U.H., M.S.H. and D.E.). 


\section{Methods}

\section{Primary rat neurons}

Hippocampi and neocortices from E19 rat embryos were dissected in ice-cold dissection media (HBBS, 1\% penicillin/streptomycin, 10 mM HEPES pH 7.3, all from Thermo Fisher Scientific), followed by enzymatic dissociation in dissection media (15 min in 0.15\% trypsin for hippocampi, 20 min in $0.25 \%$ trypsin, $0.7 \mathrm{mg} / \mathrm{ml}$ DNase I for cortices). Hippocampal neurons were plated in Neurobasal media supplemented with 2\% B27, $1 \%$ penicillin-streptomycin, $0.5 \mathrm{mM}$ L-Glutamine and $12.5 \mu \mathrm{M} \mathrm{L-}$ Glutamate (all Thermo Fisher Scientific) at 85,000 cells/ml on gold grids for cryo-ET experiments, on poly-D-lysine-coated glass coverslips (VWR) in 12-well plates for immunofluorescence, in $35 \mathrm{~mm}$ petri dishes with a $14 \mathrm{~mm}$ glass bottom inlay (MatTek Corporation) for FRAP analysis and in 6-well plates for biochemical analysis. For mass spectrometry, cortical neurons were plated in $10 \mathrm{~cm}$ petri dishes at a density of 400,000 cells/ $\mathrm{ml}$ in the above media without L-Glutamate supplementation.

\section{DNA constructs}

CDNA encoding for TDP-25 (amino acids 220-414), TDP-43 (amino acids 1-414) and NPM 1 were PCR amplified from HEK293FT (Thermo Fisher Scientific) CDNA and subsequently cloned into the FhSynW backbone (human synapsin promoter) with an N-terminal EGFP tag (May et al., 2014). TDP-43 variants with $\mathrm{N}$-terminal tagRFP were cloned in a lentiviral backbone with UbC promoter (FUW3), while human PSM B2 was expressed with a C-terminal tagRFP. TDP-43 point-mutations were introduced using standard PCR methods. The Htt97-EGFP construct was generated by subcloning the open reading frame from Addgene \#1186 into FhSynW.

\section{Lentiviral packaging and transduction}

HEK293FT cells of low passage number were seeded into three $10 \mathrm{~cm}$ dishes ( $5 \times 10^{6}$ cells/ dish) using DM EM (Thermo Fisher Scientific) supplemented with 10\% fetal bovine serum (Sigma Aldrich), 1\% penicillin-streptomycin and 1\% Non-Essential Amino Acids (Thermo Fisher Scientific) as described previously (Guo et al., 2018). On the next day, cells were co-transfected with $18.6 \mu \mathrm{g}$ transfer vector, $11 \mu \mathrm{g}$ pSPAX2 and $6.4 \mu \mathrm{g}$ pVSVG using Lipofectamine 2000 (Thermo Fisher Scientific). The transfection media was replaced by plating media supplemented with $13 \mathrm{mg} / \mathrm{mL}$ bovine serum albumin (Sigma Aldrich) on the following day. Lentivirus from the cell supernatant was collected $24 \mathrm{~h}$ later by ultracentrifugation $(87,000 \times \mathrm{g} 2 \mathrm{~h})$. Finally, lentiviral particles were resuspended in Neurobasal media and stored at $-80^{\circ} \mathrm{C}$ in aliquots.

\section{Immunofluorescence, Image Acquisition and Quantitative Analysis}

Cells grown on coverslips were briefly washed with PBS once before fixing for $10 \mathrm{~min}$ at room temperature using 4\% paraformaldehyde (Sigma Aldrich) and 4\% sucrose (Sigma-Aldrich) in PBS. Primary antibodies (anti-phospho TDP-43 [pS403/404], Cat\#TIP-PTD-P05, Cosmo Bio Co., Ltd., 1:1000; anti-Map2, Cat\#M 1406, clone AP-20, Sigma-Aldrich, 1:250) as well as secondary antibodies (Goat anti-mouse Alexa 555 or Goat anti-mouse Alexa 647, Thermo Fisher Scientific, 1:400) were diluted in GDB buffer containing $0.1 \%$ gelatin (Sigma-Aldrich), 0.3\% Triton X-100 (M erck), $450 \mathrm{mM}$ $\mathrm{NaCl}, 16 \mathrm{mM}$ sodium phosphate $\mathrm{pH}$ 7.4. Coverslips were incubated with primary antibodies 
bioRxiv preprint doi: https://doi org/10.1101/2021.03.15.435268; this version posted March 16, 2021. The copyright holder for this preprint (which was not certified by peer review) is the author/funder, who has granted bioRxiv a license to display the preprint in perpetuity. It is made available under aCC-BY-NC-ND 4.0 International license.

overnight at $4^{\circ} \mathrm{C}$ and secondary antibodies for $1 \mathrm{~h}$ at room temperature, each followed by three washes with PBS. Coverslips were mounted using Vectashield Vibrance with DAPI (Cat\# VEC-H-1800, Biozol) to counterstain nuclei.

For colocalization studies, confocal microscopy was performed using an inverted Zeiss LSM 800 Axio Observer.Z1 / 7 confocal laser scanning microscope (Carl Zeiss) with a Plan-Apochromat 63x/1.40 Oil DIC M 27 objective and equipped with the ZEN 2.5 software package (blue edition, Carl Zeiss). All images were acquired at $2048 \times 2048$ pixel resolution with 2-times averaging using two GaAsP PMT detectors at 8-bit depth.

To quantify effects of proteasomal inhibition (M G132 vs DM SO), tile scan images (>100 tiles per condition) were taken on a Leica DM i8 fluorescence microscope using a HC PL APO 40x/0,95 CORR objective, a DFC9000 camera and LAS X Software (Leica M icrosystems). Images were quantified via ImageJ/Fiji software (version 1.53c) to determine differences in GFP signal between conditions using a custom script. Integrated Density of GFP-TDP-25 was measured using a fixed threshold and the resulting median was normalized to the number of detected nuclei. DAPI-stained nuclei were counted as particles with circularity factor $0.5-1.0$ and size between $60-150 \mu \mathrm{M}^{2}$ after thresholding and binary water shedding.

\section{Cellular fractionation and immunoblotting}

Transduced neurons were washed with PBS and lysed in RIPA buffer ( $137 \mathrm{mM} \mathrm{NaCl}, 20 \mathrm{mM}$ Tris- $\mathrm{HCl}$ pH 7.5, $0.1 \%$ SDS, $10 \%$ glycerol, $1 \%$ Triton X-100, 0.5\% deoxycholate, 2 mM EDTA) freshly supplemented with $67 \mathrm{U} / \mathrm{ml}$ Benzonase Nuclease (Sigma Aldrich), protease inhibitor cocktail (Sigma Aldrich) and phosphatase inhibitor cocktail (Sigma Aldrich) on ice for $30 \mathrm{~min}$. After centrifugation $\left(18,000 \times \mathrm{g}, 30 \mathrm{~min}, 4^{\circ} \mathrm{C}\right)$, the supernatant was collected as RIPA-soluble fraction. Pellets were resuspended in $2 \%$ SDS buffer ( $2 \%$ SDS, $100 \mathrm{mM}$ Tris- $\mathrm{HCl} \mathrm{pH} \mathrm{7.0)}$ and incubated for $2 \mathrm{~h}$ at room temperature. Upon centrifugation $\left(18,000 \times \mathrm{g}, 30 \mathrm{~min}, 4^{\circ} \mathrm{C}\right)$, the supernatant was collected as SDSsoluble fraction. Samples were denatured for $10 \mathrm{~min}$ at $95^{\circ} \mathrm{C}$ after adding $3 \times$ loading buffer $(200 \mathrm{mM}$ Tris-HCl pH 6.8, 6\% SDS, 20\% glycerol, $0.1 \mathrm{~g} / \mathrm{ml}$ DTT, $0.1 \mathrm{mg}$ Bromophenol Blue). Equivalent fractions of the total cell population were loaded on $10 \%$ SDS-PAGE gels, followed by transfer to Immobilon ${ }^{\circledR}$ P PVDF membranes (M erck). M embranes were blocked in $0.2 \%$ iBlock (Thermo Fisher Scientific). The following primary antibodies were used: anti-GFP (Cat\# 75-131, clone N86/8, UC Davis/NIH Neuromab Facility) and anti-Calnexin (Cat\#ADI-SPA-860, Enzo Life Sciences).

\section{CLEM, cryo-FIB and cryo-ET}

CLEM , cryo-FIB/SEM and cryo-TEM tomographic data collection was performed as descripted in detail before (Guo et al, 2018). In brief, EM grids were mounted onto modified Autogrids sample carriers (Rigort et al, 2012) and then transferred into the cryo-stage of an FEI CorrSight microscope. Images of grid and GFP signal were respectively acquired with FEI M APS software in transmitted light and widefield mode using $5 \times$ and $20 \times$ lens. The samples were then transferred into a FIB/SEM dualbeam microscope (Quanta 3D FEG, FEI) using a cryo-transfer system (PP3000T, Quorum). Cryo-light microscope and SEM images were correlated in 2D with MAPS 2.1 software (Thermo Fisher; RRID: SCR_018738). Lamellas were prepared using $\mathrm{Ga}^{2+}$ ion beam at $30 \mathrm{kV}$ in the regions of GFP signal with final thickness of 100-200 nm.

The grids were then transferred to an FEI Titan Krios transmission electron microscope for tomographic data collection. For the whole procedure, samples were kept at liquid $\mathrm{N}_{2}$ temperature. Tomographic tilt series were recorded with a Gatan K2 Summit direct detector in counting mode. A GIF-quantum energy filter was used with a slit width of $20 \mathrm{eV}$ to remove inelastically scattered 
bioRxiv preprint doi: https://doi. org/10.1101/2021.03.15.435268; this version posted March 16, 2021. The copyright holder for this preprint (which was not certified by peer review) is the author/funder, who has granted bioRxiv a license to display the preprint in perpetuity. It is made available under aCC-BY-NC-ND 4.0 International license.

electrons. Tilt series were collected dose-symmetrically between $-51^{\circ}$ to $+69^{\circ}$ starting from $+12^{\circ}$ with an increment of $3^{\circ}$ and total dose of $110 \mathrm{e} / \AA^{2}$ using SerialEM software (M astronarde, 2005) at a pixel size of $3.52 \AA$.

\section{cET Image processing}

Image frames were aligned using M otioncor2 (Zheng et al, 2017). IM OD software package (Kremer et al, 1996) was used for tomogram reconstruction: the tilt series were firstly aligned using fiducialless patch tracking, tomogram were then reconstructed by weighted back projection of the resulting aligned images. Contrast was enhanced by filtering the tomograms using M ATLAB script tom_decov (https://github.com/dtegunov/tom_deconv).

M ATLAB with TOM toolbox(Nickell et al, 2005) was used as general platform for image processing. For segmentation, tomograms were rescaled with a binning factor of four. The membranes were firstly segmented automatically with TomoSegM emTV (M artinez-Sanchez et al, 2014) using a tensor voting method, and then manually optimized with Amira. Proteasome, ribosome and TRiC were detected using an template matching procedure with PyTOM software (Hrabe et al, 2012), templates were generated by filtering the corresponding structures from previous work (Guo et al, 2018) to $30 \AA$. The resulting coordinates were used to crop the full size subtomograms from the original tomograms, which were then CTF corrected, classified and refined using RELION (Bharat \& Scheres, 2016). In total 1170 proteasome subtomograms were picked from 11 tomograms with dominant TDP-25 aggregates for further analysis.

\section{FRAP assay}

FRAP (fluorescence recovery after photobleaching) experiments were performed on transduced hippocampal neurons on glass bottom dishes at $37^{\circ} \mathrm{C}$ in HBSS buffer (Thermo Fisher Scientific) supplemented with $20 \mathrm{mM}$ HEPES (Thermo Fisher Scientific) and $4.5 \mathrm{~g} / \mathrm{L}$ Glucose using an inverted LSM 710 Axio Observer.Z1 confocal laser scanning system (Carl Zeiss) equipped with a PlanApochromat 63×/1.40 Oil DIC M 27 objective, a PM T detector and the ZEN 2011 software (black edition, Carl Zeiss). Images were acquired with two-line averages. Three pre-bleach images were taken. Within the investigated structure (inclusion or nucleolus), a circular region of interest (ROI) of approx. $1.3 \mu \mathrm{M}$ diameter was photobleached at $100 \%$ laser power and fluorescent recovery was monitored over $75 \mathrm{~s}$ (image acquisition in $0.8 \mathrm{~s}$ intervals), $22 \mathrm{~min}$ ( $30 \mathrm{~s}$ intervals) or $450 \mathrm{~s}$ (10 s intervals). For analysis, recorded movies were aligned using the StackReg plugin of the Imagej/Fiji software. Afterwards, the mean intensity values of each region of interest (ROI) were monitored over time with ROI1 being the bleached region, ROI2 the whole nucleolus/inclusion and ROI3 the background noise. Values were then analyzed using easyFRAP (version 9.0.1) by applying the "full scale" normalization method (Rapsomaniki et al, 2012) .

\section{Immunoprecipitation}

Transduced neurons were lysed in $2 \%$ Triton X-100, $750 \mathrm{mM} \mathrm{NaCl}, 1 \mathrm{mM} \mathrm{KH}_{2} \mathrm{PO}_{4}$, freshly supplemented with $67 \mathrm{U} / \mathrm{ml}$ Benzonase Nuclease, 1x protease inhibitor cocktail and 1x phosphatase inhibitor cocktail under constant rotation for $45 \mathrm{~min}$ at $4^{\circ} \mathrm{C}$ and then centrifuged at low speed $(1,000$ $\mathrm{xg}, 5 \mathrm{~min}, 4^{\circ} \mathrm{C}$ ) to remove cellular debris but retain inclusion proteins (Hartmann et al, 2018). Supernatants were incubated with anti-GFP-antibody (Cat\# N86/38, clone N86/38, UC Davis/NIH NeuroM ab) pre-bound to Protein G Dynabeads (Cat\# 10004D, Life Technologies) on a shaking 
bioRxiv preprint doi: https://doi. org/10.1101/2021.03.15.435268; this version posted March 16, 2021. The copyright holder for this preprint (which was not certified by peer review) is the author/funder, who has granted bioRxiv a license to display the preprint in perpetuity. It is made available under aCC-BY-NC-ND 4.0 International license.

rotator for $3 \mathrm{~h}$ at $4^{\circ} \mathrm{C}$. Beads were subsequently washed three times $(150 \mathrm{mM} \mathrm{NaCl}, 50 \mathrm{mM}$ Tris- $\mathrm{HCl}$, $\mathrm{pH} 7.5$, and $5 \%$ Glycerol) and analyzed by mass spectrometry.

\section{Sample preparation for LC-MS/MS}

Beads were resuspended in $155 \mu$ denaturing buffer (8 M urea, $50 \mathrm{mM}$ Tris- $\mathrm{HCl} \mathrm{pH} \mathrm{7.5,} 1 \mathrm{mM}$ dithiothreitol). Proteins were digested off the beads with LysC ( $220 \mathrm{ng} / \mathrm{sample}$ ) for $1 \mathrm{~h}$ at room temperature while shaking at $1200 \mathrm{rpm}$. The suspension was diluted 4 -fold to lower urea concentration permitting trypsin digestion. lodoacetamide (final concentration $5 \mathrm{mM}$ ) to alkylate cysteines and trypsin ( $220 \mathrm{ng} / \mathrm{sample}$ ) were added to digest for another hour before the supernatant was transferred to another tube and digested overnight. The digest was stopped by addition of trifluoroacetic acid to $1 \% \mathrm{v} / \mathrm{v}$ and half of the peptide solution was further processed by desalting chromatography on three disks of C18 material using the STAGE-tip format (Kulak et al, 2014). Briefly, STAGE-tips were washed with $100 \mu$ l buffer B ( $50 \% \mathrm{v} / \mathrm{v}$ acetonitrile, $0.5 \% \mathrm{v} / \mathrm{v}$ acetic acid), conditioned with $100 \mu \mathrm{l}$ methanol, washed twice with $100 \mu \mathrm{l}$ buffer $\mathrm{A}(2 \% \mathrm{v} / \mathrm{v}$ acetonitrile, $0.5 \% \mathrm{v} / \mathrm{v}$ acetic acid), loaded with sample peptides, washed twice with $100 \mu$ l buffer $A$, and subjected to peptide elution by $60 \mu \mathrm{l}$ of buffer $B$. The eluate was evaporated to dryness in a vacuum concentrator. Peptides were re-suspended in $10 \mu \mathrm{l} 2 \% \mathrm{v} / \mathrm{v}$ acetonitrile, $0.5 \% \mathrm{v} / \mathrm{v}$ acetic acid, $0.1 \% \mathrm{v} / \mathrm{v}$ trifluoroacetic acid and stored at $-20^{\circ} \mathrm{C}$. Peptide concentration was measured spectrophotometrically at $280 \mathrm{~nm}$ and $500 \mathrm{ng}$ of peptide/sample were subjected to LC-M S/M S analysis.

\section{MS data acquisition}

Peptides were separated on an EASY-nanoLC 1200 HPLC system (Thermo Fisher Scientific) via inhouse packed columns (75- $\mu \mathrm{m}$ inner diameter, 50-cm length, and 1.9- $\mu \mathrm{m}$ C18 particles [Dr. M aisch $\mathrm{GmbH}]$ ) in a gradient of buffer $\mathrm{A}(0.5 \%$ formic acid) to buffer $\mathrm{B}$ ( $80 \%$ acetonitrile, $0.5 \%$ formic acid). The gradient started at $5 \% \mathrm{~B}$, increasing to $30 \% \mathrm{~B}$ in 40 minutes, further to $60 \% \mathrm{~B}$ in 4 min, to $95 \% \mathrm{~B}$ in 4 min, staying at $95 \% B$ for 4 min, decreasing to $5 \% B$ in 4 min and staying at $5 \% B$ for 4 min at a flow rate of $300 \mathrm{nl} / \mathrm{min}$ and a temperature of $60^{\circ} \mathrm{C}$. A Quadrupole Orbitrap mass spectrometer ( $Q$ Exactive HF-X; Thermo Fisher Scientific) was directly coupled to the LC via a nano-electrospray source. The $\mathrm{Q}$ Exactive HF- $x$ was operated in a data-dependent mode. The survey scan range was set from 300 to $1,650 \mathrm{~m} / \mathrm{z}$, with a resolution of 60,000 at $\mathrm{m} / \mathrm{z} 200$. Up to the 12 most abundant isotope patterns with a charge of two to five were isolated and subjected to collision-induced dissociation fragmentation at a normalized collision energy of 27 , an isolation window of $1.4 \mathrm{Th}$, and a M S/MS resolution of 15,000 at $\mathrm{m} / \mathrm{z} 200$. Dynamic exclusion to minimize re-sequencing was set to $30 \mathrm{~s}$.

\section{MS raw data processing}

To process MS raw files, we employed the MaxQuant software version 1.6.0.15 (Cox \& M ann, 2008), searching against the UniProtKB rat FASTA database using canonical and isoform protein sequences downloaded in M arch 2018. Default search parameters were utilized unless stated differently. In brief, tryptic peptides with a minimum length of 7 amino acids, a maximum mass of $4600 \mathrm{Da}$, and two miscleavages at maximum were searched. Carbamidomethlyation was set as a fixed modification and methionine oxidation and protein $\mathrm{N}$-terminal acetylation as variable modifications. A maximum of five modifications per peptide was permitted. A false discovery rate (FDR) cutoff of $1 \%$ was applied at the peptide and protein level. The search feature "M atch between runs," which allows the transfer of peptide identifications in the absence of MS/MS-based identification after 
nonlinear retention time alignment was enabled with a maximum retention time window of 0.7 min. Protein abundances were normalized with the M axLFQ label-free normalization algorithm built into MaxQuant (Cox et al, 2014).

\section{Bioinformatic data analysis}

The dataset was further processed and analyzed in the Perseus environment version 1.6.1.3 (Tyanova et al, 2016). Reverse (decoy) hits, proteins only identified by site, potential contaminants, and proteins not quantified in at least $65 \%$ of samples of at least one condition (construct and DIV) were removed. Protein abundances were log2-transformed. M issing values for protein abundances were imputed from a normal distribution around the detection limit with a standard deviation of 0.3 times that of the quantified protein abundance distribution combining all samples and a downshift of 1.8 standard deviations. Volcano plot data were generated with the built-in Perseus tool using a SAM statistic with an s0-paramater of 0.1 to integrate the effect size and a permutation-based FDR control set to $5 \%$ (Tusher et al, 2001). Proteins significantly (q-value $<5 \%$ ) associated with TDP-25 or TDP-43 were termed interactors and included in the further analysis.

To analyze the content of low-complexity regions of neuronal TDP-25/TDP-43 interactors, we automatically queried the D2P2 database based on M axQuant reported UniProt identifier, using the first entry if multiple identifiers were reported in protein groups. Low complexity regions were defined as regions with a consensus of at least 6 of 9 analysis pipelines within D2P2 (Oates et al, 2013). Gene ontology (GO) analysis was performed focusing on the "biological process" categories of differentially immunoprecipitated proteins using clusterProfiler version 3.12 (Yu et al, 2012). A manual selection is shown to best represent the key pathways. The full list of significant GO terms is shown in Tables S2. The MS proteomics data have been deposited to the ProteomeXchange Consortium via the PRIDE partner repository with the dataset identifier PXD024358.

\section{Flow Cytometry}

Flow cytometry experiments were performed as described before (Guo et al, 2018). UbG76V-GFP proteasome reporter cells were dissociated $72 \mathrm{~h}$ after transfection and analyzed with a Thermo Fisher Attune NxT Flow Cytometer (Thermo Fisher Scientific). To compensate for crosstalk between GFP and tagRFP, HEK293 were transfected with tagRFP constructs only. Raw flow cytometry data were analyzed using FlowJ o software (version 9.9, Treestar). The tagRFP channel was subdivided into different gates, corresponding to the log of fluorescence intensity of the transfected protein.

\section{Data Visualization and Statistical Analysis}

Data were visualized and statistically analyzed using $R$ (version 3.6.1). Assumptions for parametric tests were analyzed with Shapiro-Wilk test and Levene's test. Kruskal-Wallis test and subsequent Pairwise Wilcoxon Rank Sum Tests with Benjamini-Hochberg correction were used as nonparametric tests. Data are plotted as mean \pm standard deviation (SD) or box plots/violin plots. In the legends mean $\pm 95 \% \mathrm{Cl}$ is noted. 


\section{References}

Asano S, Fukuda Y, Beck F, Aufderheide A, Forster F, Danev R, Baumeister W (2015) Proteasomes. A molecular census of $26 \mathrm{~S}$ proteasomes in intact neurons. Science 347: 439-442

Bauerlein FJB, Saha I, M ishra A, Kalemanov M, Martinez-Sanchez A, Klein R, Dudanova I, Hipp MS, Hartl FU, Baumeister W et al (2017) In Situ Architecture and Cellular Interactions of PolyQ Inclusions. Cell 171: 179-187 e110

Bax M, M cKenna J, Do-Ha D, Stevens CH, Higginbottom S, Balez R, Cabral-da-Silva M EC, Farrawell NE, Engel M , Poronnik P et al (2019) The Ubiquitin Proteasome System Is a Key Regulator of Pluripotent Stem Cell Survival and M otor Neuron Differentiation. Cells 8

Bett JS, Cook C, Petrucelli L, Bates GP (2009) The ubiquitin-proteasome reporter GFPu does not accumulate in neurons of the R6/2 transgenic mouse model of Huntington's disease. PLoS One 4: e5128

Bharat TA, Scheres SH (2016) Resolving macromolecular structures from electron cryo-tomography data using subtomogram averaging in RELION. Nat Protoc 11: 2054-2065

Blokhuis AM, Koppers M, Groen EJN, van den Heuvel DM A, Dini Modigliani S, Anink JJ, Fumoto K, van Diggelen F, Snelting A, Sodaar P et al (2016) Comparative interactomics analysis of different ALSassociated proteins identifies converging molecular pathways. Acta Neuropathol 132: 175-196

Cao Q, Boyer DR, Sawaya M R, Ge P, Eisenberg DS (2019) Cryo-EM structures of four polymorphic TDP-43 amyloid cores. Nat Struct M ol Biol 26: 619-627

Cassel JA, Reitz AB (2013) Ubiquilin-2 (UBQLN2) binds with high affinity to the C-terminal region of TDP-43 and modulates TDP-43 levels in $\mathrm{H} 4$ cells: characterization of inhibition by nucleic acids and 4aminoquinolines. Biochim Biophys Acta 1834: 964-971

Chou CC, Zhang Y, Umoh M E, Vaughan SW, Lorenzini I, Liu F, Sayegh M, Donlin-Asp PG, Chen YH, Duong DM et al (2018) TDP-43 pathology disrupts nuclear pore complexes and nucleocytoplasmic transport in ALS/FTD. Nat Neurosci 21: 228-239

Collins GA, Goldberg AL (2017) The Logic of the 26S Proteasome. Cell 169: 792-806

Conicella AE, Zerze GH, M ittal J, Fawzi NL (2016) ALS M utations Disrupt Phase Separation Mediated by alpha-Helical Structure in the TDP-43 Low-Complexity C-Terminal Domain. Structure 24: 15371549

Cox J, Hein MY, Luber CA, Paron I, Nagaraj N, M ann M (2014) Accurate proteome-wide label-free quantification by delayed normalization and maximal peptide ratio extraction, termed MaxLFQ. Mol Cell Proteomics 13: 2513-2526 
Cox J, M ann M (2008) MaxQuant enables high peptide identification rates, individualized p.p.b.range mass accuracies and proteome-wide protein quantification. Nat Biotechnol 26: 1367-1372

Dantuma NP, Lindsten K, Glas R, Jellne M, M asucci M G (2000) Short-lived green fluorescent proteins for quantifying ubiquitin/proteasome-dependent proteolysis in living cells. Nat Biotechnol 18: 538543

Deng HX, Chen W, Hong ST, Boycott KM, Gorrie GH, Siddique N, Yang Y, Fecto F, Shi Y, Zhai H et al (2011) Mutations in UBQLN2 cause dominant X-linked juvenile and adult-onset ALS and ALS/ dementia. Nature 477: 211-215

Eisenberg D, Jucker M (2012) The amyloid state of proteins in human diseases. Cell 148: 1188-1203

Feneberg E, Gordon D, Thompson AG, Finelli MJ, Dafinca R, Candalija A, Charles PD, M ager I, Wood MJ, Fischer $R$ et al (2020) An ALS-linked mutation in TDP-43 disrupts normal protein interactions in the motor neuron response to oxidative stress. Neurobiol Dis 144: 105050

Freibaum BD, Chitta RK, High AA, Taylor JP (2010) Global analysis of TDP-43 interacting proteins reveals strong association with RNA splicing and translation machinery. J Proteome Res 9: 1104-1120

Frottin F, Schueder F, Tiwary S, Gupta R, Korner R, Schlichthaerle T, Cox J, Jungmann R, Hartl FU, Hipp MS (2019) The nucleolus functions as a phase-separated protein quality control compartment. Science 365: 342-347

Gao FB, Almeida S, Lopez-Gonzalez R (2017) Dysregulated molecular pathways in amyotrophic lateral sclerosis-frontotemporal dementia spectrum disorder. EM BO J 36: 2931-2950

Gasset-Rosa F, Lu S, Yu H, Chen C, M elamed Z, Guo L, Shorter J, Da Cruz S, Cleveland DW (2019) Cytoplasmic TDP-43 De-mixing Independent of Stress Granules Drives Inhibition of Nuclear Import, Loss of Nuclear TDP-43, and Cell Death. Neuron 102: 339-357 e337

Gitcho M A, Strider J, Carter D, Taylor-Reinwald L, Forman MS, Goate AM , Cairns NJ (2009) VCP mutations causing frontotemporal lobar degeneration disrupt localization of TDP-43 and induce cell death. J Biol Chem 284: 12384-12398

Gomes E, Shorter J (2019) The molecular language of membraneless organelles. J Biol Chem 294: 7115-7127

Guenther EL, Cao Q, Trinh H, Lu J, Sawaya M R, Cascio D, Boyer DR, Rodriguez JA, Hughes M P, Eisenberg DS (2018) Atomic structures of TDP-43 LCD segments and insights into reversible or pathogenic aggregation. Nat Struct M ol Biol 25: 463-471 
Guo Q, Lehmer C, M artinez-Sanchez A, Rudack T, Beck F, Hartmann H, Perez-Berlanga M, Frottin F, Hipp MS, Hartl FU et al (2018) In Situ Structure of Neuronal C9orf72 Poly-GA Aggregates Reveals Proteasome Recruitment. Cell 172: 696-705 e612

Hasegawa M, Arai T, Nonaka T, Kametani F, Yoshida M, Hashizume Y, Beach TG, Buratti E, Baralle F, M orita $M$ et al (2008) Phosphorylated TDP-43 in frontotemporal lobar degeneration and amyotrophic lateral sclerosis. Ann Neurol 64: 60-70

Hipp MS, Kasturi P, Hartl FU (2019) The proteostasis network and its decline in ageing. Nature reviews Molecular cell biology 20: 421-435

Hrabe T, Chen Y, Pfeffer S, Cuellar LK, M angold AV, Forster F (2012) PyTom: a python-based toolbox for localization of macromolecules in cryo-electron tomograms and subtomogram analysis. J Struct Biol 178: 177-188

Igaz LM, Kwong LK, Xu Y, Truax AC, Uryu K, Neumann M, Clark CM, Elman LB, M iller BL, Grossman M et al (2008) Enrichment of C-terminal fragments in TAR DNA-binding protein-43 cytoplasmic inclusions in brain but not in spinal cord of frontotemporal lobar degeneration and amyotrophic lateral sclerosis. Am J Pathol 173: 182-194

Jain S, Wheeler JR, Walters RW, Agrawal A, Barsic A, Parker R (2016) ATPase-M odulated Stress Granules Contain a Diverse Proteome and Substructure. Cell 164: 487-498

Kato M, Han TW, Xie S, Shi K, Du X, Wu LC, M irzaei H, Goldsmith EJ, Longgood J, Pei J et al (2012) Cell-free formation of RNA granules: low complexity sequence domains form dynamic fibers within hydrogels. Cell 149: 753-767

Kremer JR, M astronarde DN, M cIntosh JR (1996) Computer visualization of three-dimensional image data using IM OD. J Struct Biol 116: 71-76

Kulak NA, Pichler G, Paron I, Nagaraj N, M ann M (2014) M inimal, encapsulated proteomic-sample processing applied to copy-number estimation in eukaryotic cells. Nat M ethods 11: 319-324

Ling SC, Albuquerque CP, Han JS, Lagier-Tourenne C, Tokunaga S, Zhou H, Cleveland DW (2010) ALSassociated mutations in TDP-43 increase its stability and promote TDP-43 complexes with FUS/TLS. Proc Natl Acad Sci U S A 107: 13318-13323

Mackenzie IR, Arzberger T, Kremmer E, Troost D, Lorenzl S, M ori K, Weng SM, Haass C, Kretzschmar HA, Edbauer D et al (2013) Dipeptide repeat protein pathology in C9ORF72 mutation cases: clinicopathological correlations. Acta Neuropathol 126: 859-879

Mann JR, Gleixner AM, M auna JC, Gomes E, DeChellis-M arks M R, Needham PG, Copley KE, Hurtle B, Portz B, Pyles NJ et al (2019) RNA Binding Antagonizes Neurotoxic Phase Transitions of TDP-43.

Neuron 102: 321-338 e328 
M artin EW, M ittag T (2018) Relationship of Sequence and Phase Separation in Protein Low-

Complexity Regions. Biochemistry 57: 2478-2487

Martinez-Sanchez A, Garcia I, Asano S, Lucic V, Fernandez JJ (2014) Robust membrane detection based on tensor voting for electron tomography. J Struct Biol 186: 49-61

Mastronarde DN (2005) Automated electron microscope tomography using robust prediction of specimen movements. J Struct Biol 152: 36-51

M urray DT, Kato M, Lin Y, Thurber KR, Hung I, M cKnight SL, Tycko R (2017) Structure of FUS Protein Fibrils and Its Relevance to Self-Assembly and Phase Separation of Low-Complexity Domains. Cell 171: 615-627 e616

Nickell S, Forster F, Linaroudis A, Net WD, Beck F, Hegerl R, Baumeister W, Plitzko JM (2005) TOM software toolbox: acquisition and analysis for electron tomography. J Struct Biol 149: 227-234

Oates M E, Romero P, Ishida T, Ghalwash M, M izianty MJ, Xue B, Dosztanyi Z, Uversky VN, Obradovic Z, Kurgan L et al (2013) D(2)P(2): database of disordered protein predictions. Nucleic Acids Res 41: D508-516

Olzscha H, Schermann SM, Woerner AC, Pinkert S, Hecht M H, Tartaglia GG, Vendruscolo M, HayerHartl M, Hartl FU, Vabulas RM (2011) Amyloid-like aggregates sequester numerous metastable proteins with essential cellular functions. Cell 144: 67-78

Patel A, Lee HO, Jawerth L, Maharana S, Jahnel M, Hein MY, Stoynov S, M ahamid J, Saha S, Franzmann TM et al (2015) A Liquid-to-Solid Phase Transition of the ALS Protein FUS Accelerated by Disease M utation. Cell 162: 1066-1077

Prasad A, Bharathi V, Sivalingam V, Girdhar A, Patel BK (2019) M olecular M echanisms of TDP-43 Misfolding and Pathology in Amyotrophic Lateral Sclerosis. Front M ol Neurosci 12: 25

Qamar S, Wang G, Randle SJ, Ruggeri FS, Varela JA, Lin JQ, Phillips EC, Miyashita A, Williams D, Strohl F et al (2018) FUS Phase Separation Is M odulated by a M olecular Chaperone and M ethylation of Arginine Cation-pi Interactions. Cell 173: 720-734 e715

Rapsomaniki M A, Kotsantis P, Symeonidou IE, Giakoumakis NN, Taraviras S, Lygerou Z (2012) easyFRAP: an interactive, easy-to-use tool for qualitative and quantitative analysis of FRAP data. Bioinformatics 28: 1800-1801

Rigort A, Bauerlein FJ, Villa E, Eibauer M, Laugks T, Baumeister W, Plitzko JM (2012) Focused ion beam micromachining of eukaryotic cells for cryoelectron tomography. Proc Natl Acad Sci U SA 109: 4449-4454 
Schaefer T, Riera-Tur I, Hornburg D, M ishra A, Fernández-M osquera L, Raimundo N, Mann M, Baumeister W, Klein R, M eissner F et al (2019) Amyloid-like aggregates cause lysosomal defects in neurons via gain-of-function toxicity. bioRxiv: 2019.2012.2016.877431

Scotter EL, Vance C, Nishimura AL, Lee YB, Chen HJ, Urwin H, Sardone V, Mitchell JC, Rogelj B, Rubinsztein DC et al (2014) Differential roles of the ubiquitin proteasome system and autophagy in the clearance of soluble and aggregated TDP-43 species. J Cell Sci 127: 1263-1278

Tashiro Y, Urushitani M, Inoue H, Koike M, Uchiyama Y, Komatsu M, Tanaka K, Yamazaki M, Abe M, $\mathrm{M}$ isawa $\mathrm{H}$ et al (2012) M otor neuron-specific disruption of proteasomes, but not autophagy, replicates amyotrophic lateral sclerosis. J Biol Chem 287: 42984-42994

Trinkaus VA, Riera-Tur I, M artínez-Sánchez A, Bäuerlein FJB, Guo Q, Arzberger T, Baumeister W, Dudanova I, Hipp MS, Hartl FU et al (2020) <em>ln situ $<$ em>architecture of neuronal $\alpha$-Synuclein inclusions. bioRxiv: 2020.2008.2007.234138

Tusher VG, Tibshirani R, Chu G (2001) Significance analysis of microarrays applied to the ionizing radiation response. Proc Natl Acad Sci U S A 98: 5116-5121

Tyanova S, Temu T, Sinitcyn P, Carlson A, Hein MY, Geiger T, Mann M , Cox J (2016) The Perseus computational platform for comprehensive analysis of (prote)omics data. Nat M ethods 13: 731-740

Vogler TO, Wheeler JR, Nguyen ED, Hughes M P, Britson KA, Lester E, Rao B, Betta ND, Whitney ON, Ewachiw TE et al (2018) TDP-43 and RNA form amyloid-like myo-granules in regenerating muscle. Nature 563: 508-513

Walker AK, Tripathy K, Restrepo CR, Ge G, Xu Y, Kwong LK, Trojanowski JQ, Lee VM (2015) An insoluble frontotemporal lobar degeneration-associated TDP-43 C-terminal fragment causes neurodegeneration and hippocampus pathology in transgenic mice. Hum M ol Genet 24: 7241-7254

Watabe K, Kato Y, Sakuma M, M urata M, Niida-Kawaguchi M, Takemura T, Hanagata N, Tada M, Kakita A, Shibata N (2020) Praja1 RING-finger E3 ubiquitin ligase suppresses neuronal cytoplasmic TDP-43 aggregate formation. Neuropathology

Yu G, Wang LG, Han Y, He QY (2012) clusterProfiler: an R package for comparing biological themes among gene clusters. OMICS 16: 284-287

Yu H, Lu S, Gasior K, Singh D, Vazquez-Sanchez S, Tapia O, Toprani D, Beccari M S, Yates JR, 3rd, Da Cruz S et al (2021) HSP70 chaperones RNA-free TDP-43 into anisotropic intranuclear liquid spherical shells. Science 371

Zhang YJ, Xu YF, Cook C, Gendron TF, Roettges P, Link CD, Lin WL, Tong J, Castanedes-Casey M, Ash P et al (2009) Aberrant cleavage of TDP-43 enhances aggregation and cellular toxicity. Proc Natl Acad Sci U SA 106: 7607-7612 
bioRxiv preprint doi: https://doi.org/10.1101/2021.03.15.435268; this version posted March 16, 2021. The copyright holder for this preprint (which was not certified by peer review) is the author/funder, who has granted bioRxiv a license to display the preprint in perpetuity. It is made available under aCC-BY-NC-ND 4.0 International license.

Zheng SQ, Palovcak E, Armache JP, Verba KA, Cheng Y, Agard DA (2017) MotionCor2: anisotropic correction of beam-induced motion for improved cryo-electron microscopy. Nat M ethods 14: 331332 
A

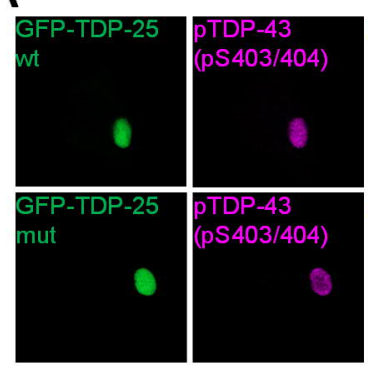

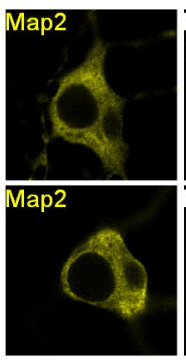

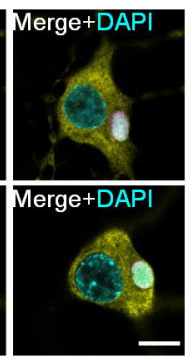

B

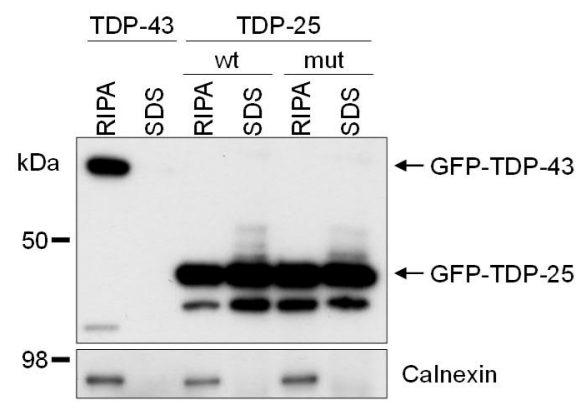

C

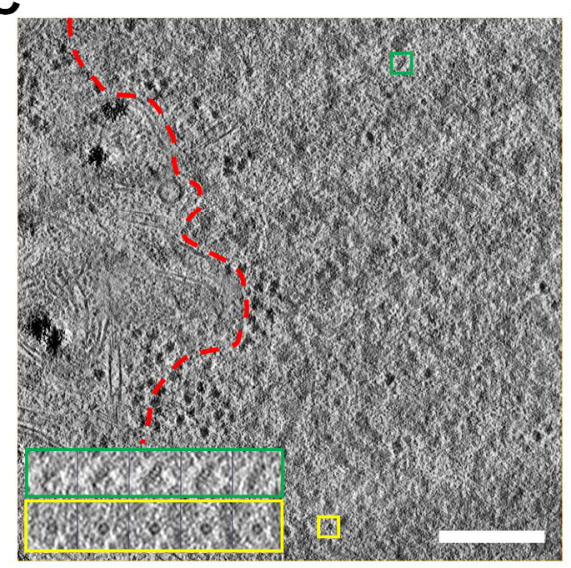

\section{D}
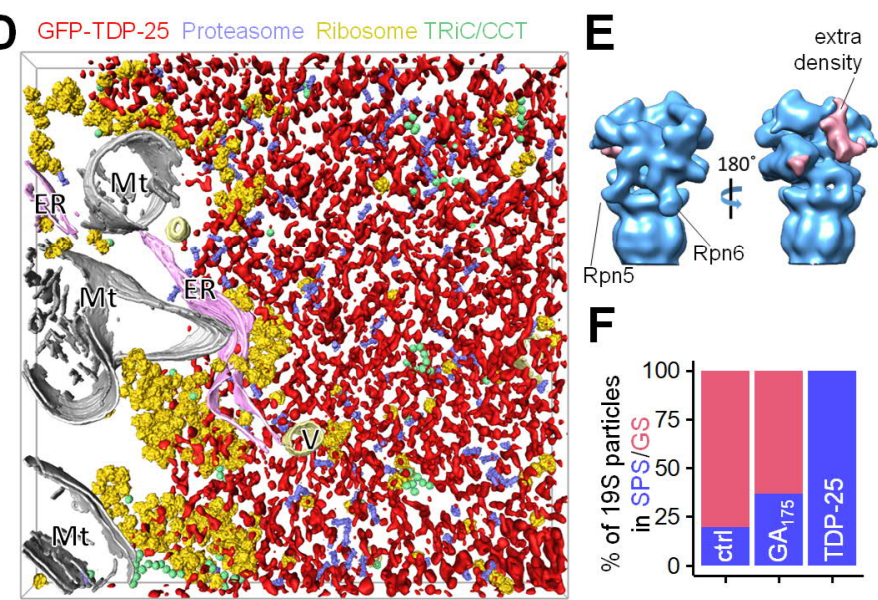

\section{Figure 1: TDP-25 forms amorphous inclusions enriched in proteasomes.}

A: Primary rat hippocampal neurons were transduced with constructs expressing GFP-tagged TDP-25 (amino acids 220-414 of TDP-43) on day 5 in vitro and cultured for 8 additional days (DIV5+8). Wildtype or variant containing 8 ALS-causing mutations. Immunofluorescence shows disease-related phosphorylation at serine 403/404. Counterstain to label the neuronal cytoskeleton (M AP2) and nuclei (DAPI). Scale bar $=10 \mu \mathrm{M}$.

B: Hippocampal neurons transduced with GFP-TDP-25 constructs (DIV5+8) or GFP-TDP-43 (DIV5+4 due to higher toxicity) were analyzed by sequential extraction in RIPA buffer followed by $2 \%$ SDS. Immunoblots for GFP and loading control Calnexin.

C: Tomographic slice of an aggregate within a GFP-TDP-25 wild-type transduced neuron (DIV5+8). Colored boxes show a series of higher magnification tomographic slices of representative protein complexes detected in the tomogram. Green boxes show side views of single-capped, yellow boxes show smaller ring-like structures of $26 \mathrm{~S}$ proteasomes. Red dotted line segments aggregate area. Scale bar $=200 \mathrm{~nm}$.

$D: 3 D$ rendering of the aggregate shown in (C). Amorphous aggregate material is labeled in red, proteasomes in violet, ribosomes in yellow, TRiC/CCT chaperones in green, mitochondria (Mt) in white, ER in pink and other vesicles (V) in light yellow. Compare M ovie S1.

E: Subtomogram averaging of macromolecules in GFP-TDP-25 inclusions reveals the proteasome structure at $\sim 20 \AA$ resolution (see Fig. S1). The positions of Rpn5/PSM D11 and Rpn6/PSDM 12 are indicated. Prominent extra densities in the substrate binding region are colored in pink in the 3D rendering.

F: Classification based on the conformation of the regulatory particle in TDP-25 inclusions compared to non-transduced control neurons (Asano et al, 2015) and poly-GA inclusions (Guo et al, 2018). GS = Ground State, SPS = Substrate Processing State. 

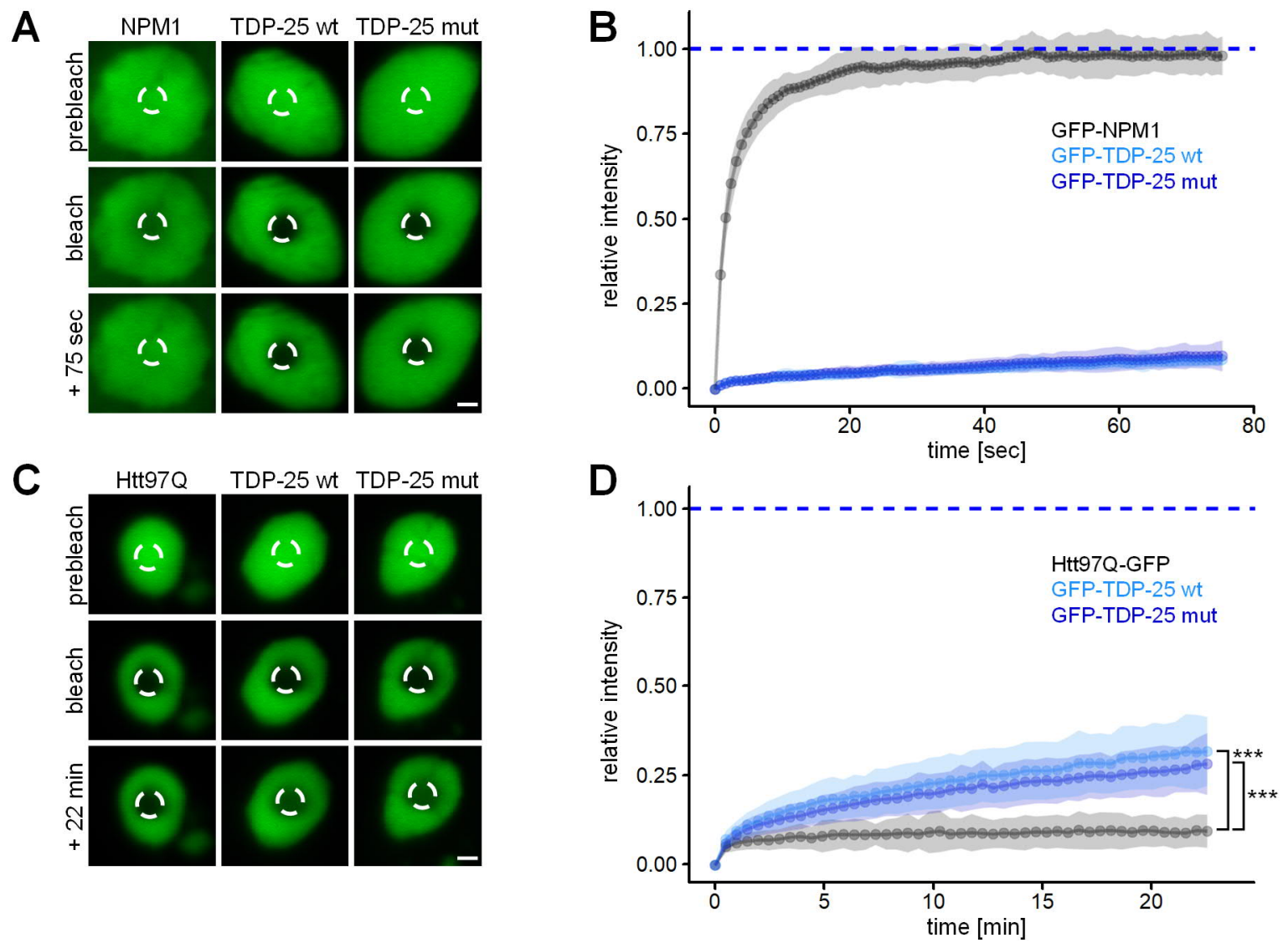

\section{Figure 2: TDP-25 inclusions are neither liquid-like nor solid-like.}

Primary rat hippocampal neurons were transduced with GFP-TDP-25 variants, GFP-NPM 1 or Htt97QGFP and analyzed by fluorescence recovery after photobleaching (FRAP) at DIV5+8, except Htt97QGFP which was analyzed at DIV $5+6$ to avoid excessive toxicity. Example images with indicated bleach regions (dashed circles) are shown $(A, C)$. Scale bar $=1.5 \mu \mathrm{M}$. Resulting normalized FRAP curves (relative fluorescence intensity over time) with values representing means $\pm S D$ are shown in $(B, D)$. (B) GFP-TDP-25 wild-type ( $n=35$ cells), GFP-TDP-25 mutant ( $n=38$ cells), GFP-NPM 1 ( $n=37$ cells) out of three independent experiments. In (D), the recovery fraction averaged at $>20$ min timepoints was compared from four independent experiments. Htt97Q-GFP ( $n=25$ cells) vs GFP-TDP-25 wt ( $n=23$ ) vs GFP-TDP-25 mut $(n=24): H(1)=46.376, d f=2, p \varangle 0.001$, Kruskal-Wallis Test. Htt97Q-GFP vs GFP-TDP-25 wt $p=1.9 \times 10^{-11}$, Htt97Q-GFP vs GFP-TDP-25 mut $p=4.3 \times 10^{-12}$, Pairwise Wilcoxon Rank Sum Tests with Benjamini-Hochberg correction. 

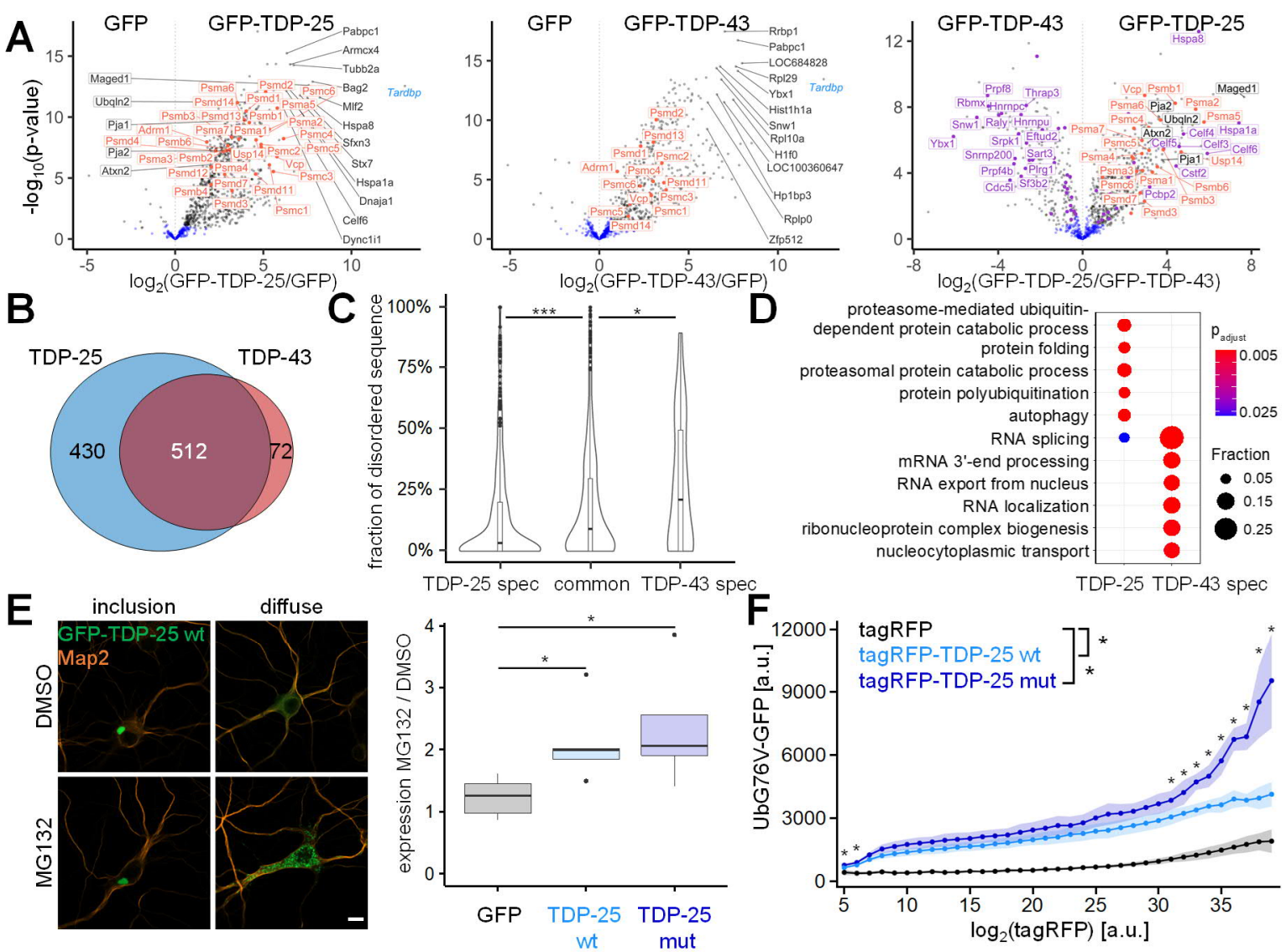

Figure 3: TDP-25 sequesters and inhibits the proteasome.

A: Rat primary cortical neurons were transduced with GFP-TDP-25 wild-type, GFP-TDP-43 or GFP at DIV5. Due to high toxicity, GFP-TDP-43 $(n=6)$ and GFP control samples $(n=6)$ were harvested at DIV5+4, while GFP-TDP-25 $(n=5)$ and additional GFP control samples $(n=6)$ were harvested at DIV5+8. Immunoprecipitates were analyzed by LC-M S/M S. Volcano plots indicate enrichment and statistical significance (grey dots: FDR-corrected $p \varangle 0.05$ ). Proteins associated with $\mathrm{GO}$ term "proteasome complex" (G0:0000502) are labeled in orange and proteins associated with GO term "RNA splicing" (G0:0008380) in purple. Full data is list in Table S1. A comparison with previous proteomic studies is shown in Fig. S3A.

B: Overlap of GFP-TDP-25 and GFP-TDP-43 interactomes. Significant interactors in FDR-based approach (see methods).

C: Combined violin and box plot of the proportion of low-complexity regions (D2P2) of GFP-TDP-25specific, GFP-TDP-43-specific or shared/common interactors. TDP-25 specific ( $n=432$ Uniprot IDs) vs TDP-43 specific ( $n=78$ ) vs common interactors $(n=481)$ : $H(1)=22.276, d f=2, p<0.001$, Kruskal-Wallis test. TDP-25 specific vs TDP-43 specific: $p=0.00054$, TDP-25 specific vs common: $p=0.00035$, TDP-43 specific vs common interactors: $p=0.04893$, Pairwise Wilcoxon Rank Sum Tests with BenjaminiHochberg correction.

D: Gene ontology (GO) enrichment analysis (biological process) in interactors specific for either GFPTDP-25 or GFP-TDP-43. Full list of GO terms is found in Table S2.

E: Immunofluorescence of hippocampal neurons transduced with GFP, GFP-TDP-25 wild-type or mutant and treated with M G132 (10 $\mu \mathrm{M}, 14 \mathrm{~h}$, DIV5+8). Representative images of GFP-TDP-25 
inclusions and diffuse cytoplasmic staining. Automated quantification of GFP expression normalized to DAPI stained nuclei (not shown) in M G132 treated cells compared to DM SO control. Counterstain to label the neuronal cytoskeleton (M AP2). Scale bar $=10 \mu M$. GFP ( $=5$ independent experiments) vs GFP-TDP-25 wt $(n=5)$ vs GFP-TDP-25 mut $(n=5): H(1)=7.34, d f=2, p \varangle 0.05$, Kruskal-Wallis test. GFP vs GFP-TDP-25 wt: $p=0.048$, GFP vs GFP-TDP-25 mut: $p=0.048$, Pairwise Wilcoxon Rank Sum Tests with Benjamini-Hochberg correction.

F: A HEK293 cell line expressing the proteostasis reporter UbG76V-GFP was transiently transfected with RFP, RFP-TDP-25 wild-type or mutant. UbG76V-GFP levels were analyzed by flow cytometry 72 $\mathrm{h}$ after transfection. The relationship of binned tagRFP fluorescence and UbG76V-GFP fluorescence shows a concentration-dependent accumulation of UbG76V-GFP in cells expressing RFP-TDP-25 wildtype and even more so in the mutant variant. Values shown are means $\pm S D$ obtained from $n=4$ independent experiments. Pairwise comparison using Wilcoxon rank sum test with BenjaminiHochberg correction was performed for each tagRFP intensity gate respectively. Adjusted $p$-values for comparison of wild-type and mutant TDP- 25 are indicated by asterisks: $*$ denotes $p \varangle 0.05$. tagRFP-TDP-25 wild-type and mutant showed statistically significant differences vs tagRFP control in all gates. Reporter levels were significantly higher for TDP-25 mutant than wild-type in highexpressing cells. 


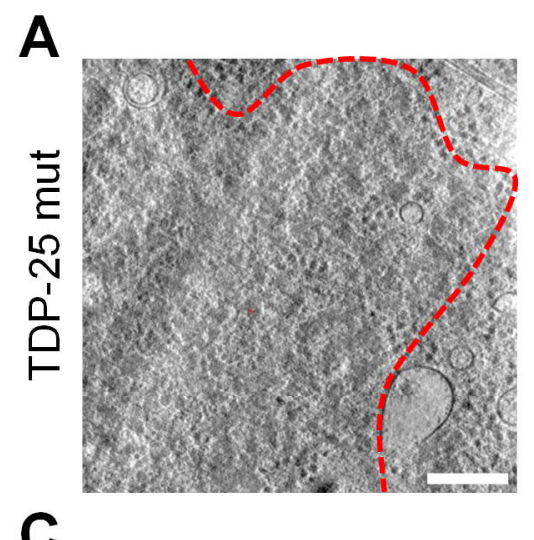

C

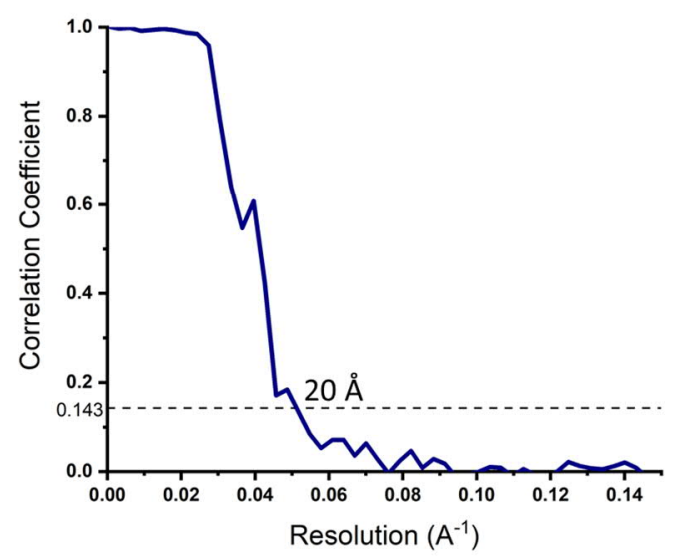

B

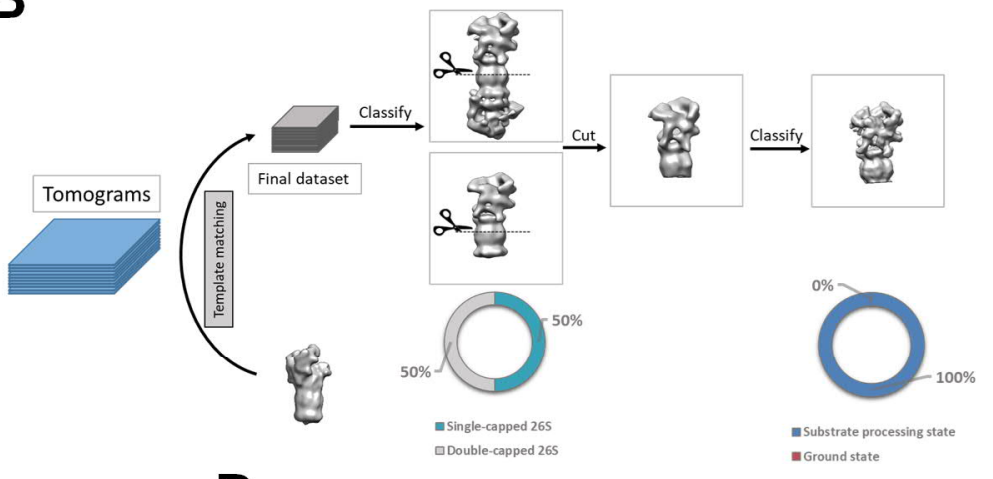

D

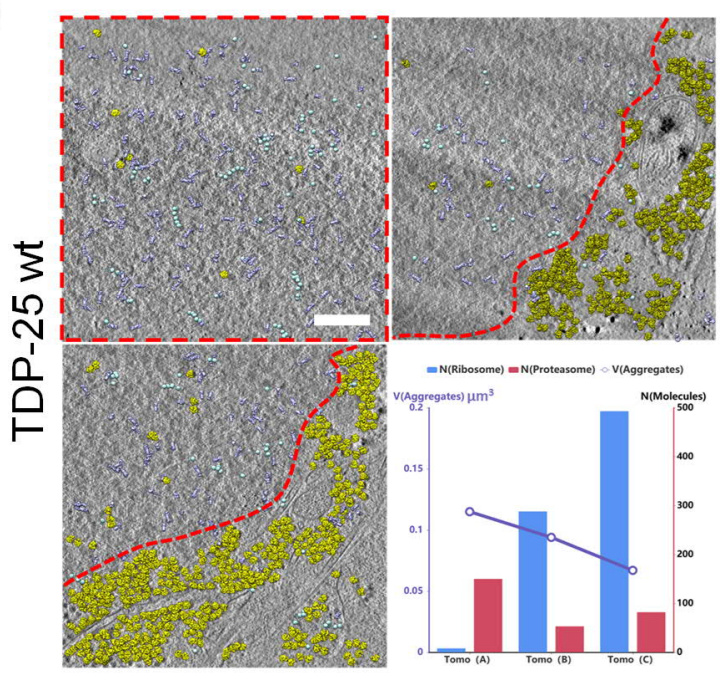

Figure S1: Proteasomes are enriched in TDP-25 inclusions.

A: Tomographic slice of an aggregate within a GFP-TDP-25 mutant transduced neuron (DIV5+8). Red dotted line segments aggregate area. Scale bar $=200 \mathrm{~nm}$.

B: Workflow of subtomogram averaging and classification. Subtomograms were identified using a low-resolution single-capped proteasome as template. All the proteasomes were firstly classified into single-capped or double-capped. To further analyze the conformation of the regulatory particles, all the proteasomes were cut in silico between the $\beta$-rings of the core particle, resulting in two independent particles for double-capped ones. Cut out regulatory particles were merged and subjected to a further round of classification.

C: Gold-standard Fourier shell correlation curve of the proteasome structure show a resolution of $20 \AA$.

D: Molecular mapping in three samples of GFP-TDP-25 wild-type inclusions in transduced neurons (DIV5+8). Regions containing GFP-TDP-25 are outlined in red. For the whole tomogram, proteasomes (purple), TRiC (cyan) and ribosomes (yellow) are mapped to their original positions and orientations using the information from template matching and subtomogram averaging. The numbers of proteasomes and ribosomes detected in the tomograms plotted versus the volume of the region containing GFP-TDP-25 inclusions. Scale bar $=200 \mathrm{~nm}$. 


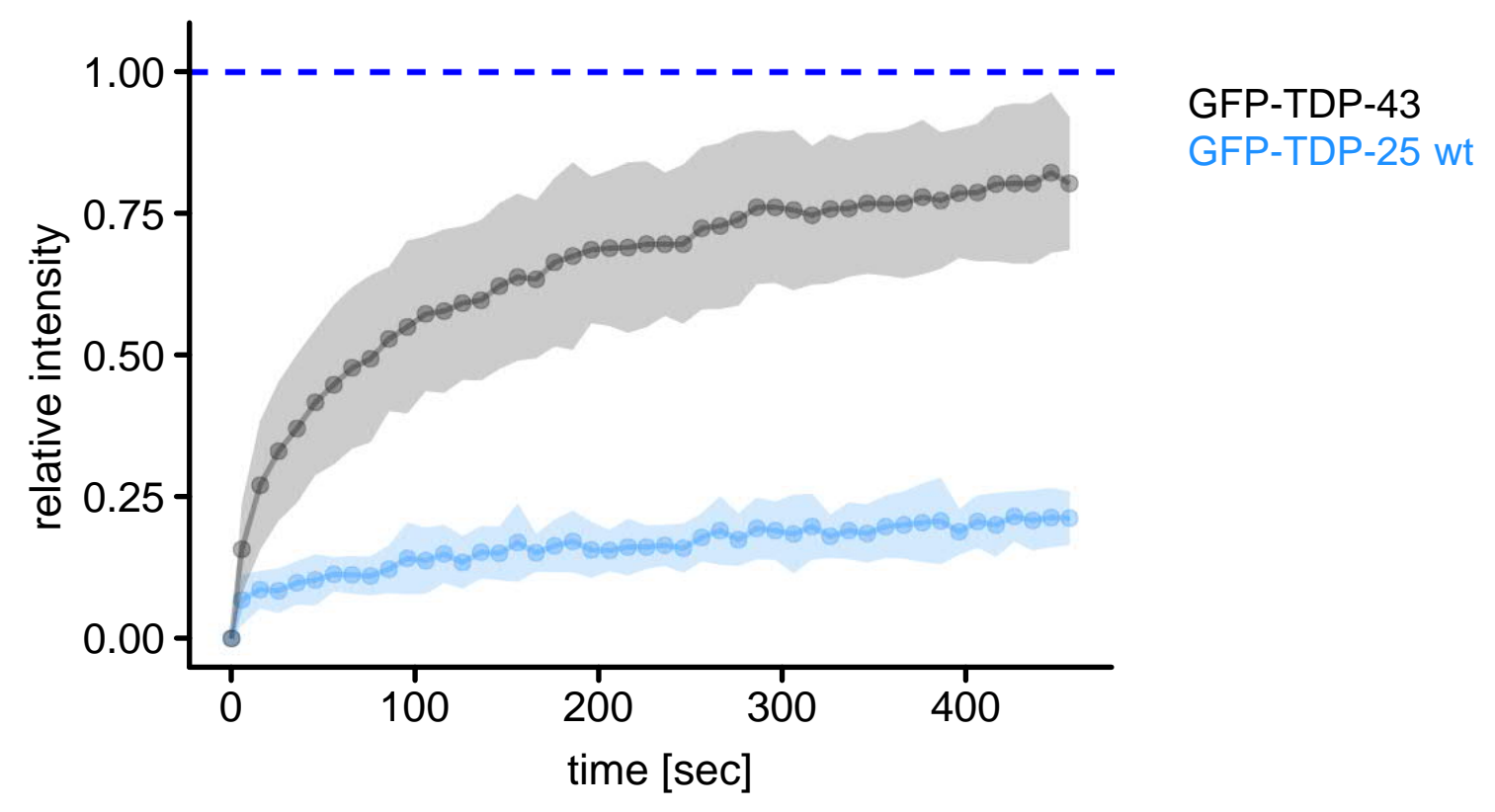

Figure S2: TDP-25 is less mobile than TDP-43.

Rat primary hippocampal neurons were transduced with GFP-TDP-25 (DIV5+8) or GFP-TDP-43 (DIV9+4) and analyzed by FRAP. Resulting normalized FRAP curves (relative fluorescence intensity over time) with values representing means \pm SD are shown. GFP-TDP- 25 wild-type ( $n=18$ cells), GFPTDP-43 ( $n=20$ cells) from three independent experiments. 
A

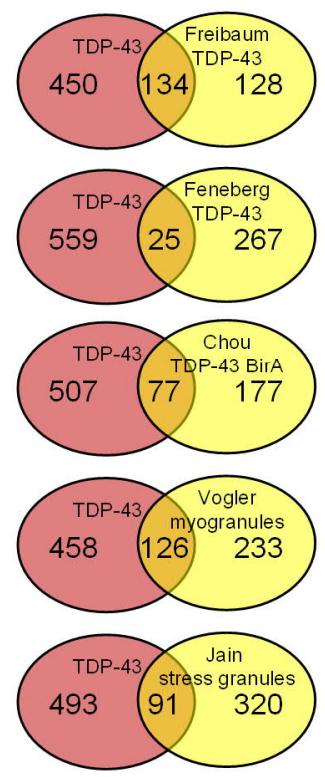

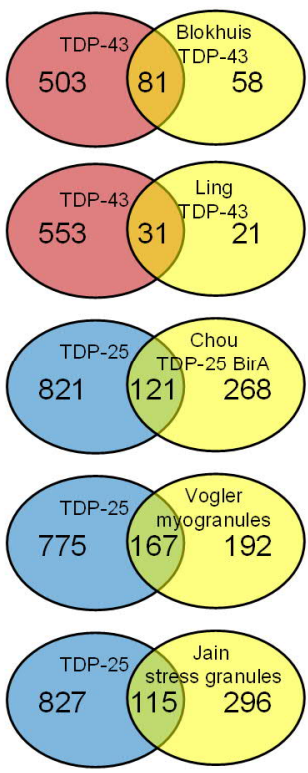

B

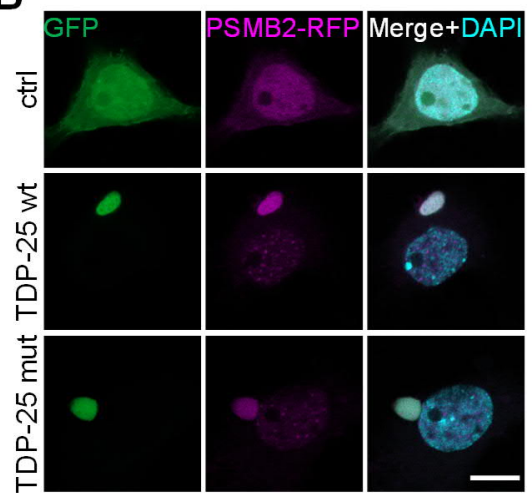

Figure S3: Overlap of TDP-43 and TDP-25 interactome with published datasets.

A: Proteins identified in the GFP-TDP-43 interactome (uniquely or in a cluster) were compared to published datasets (Blokhuis et al, 2016; Chou et al, 2018; Feneberg et al, 2020; Freibaum et al, 2010; Ling et al, 2010). LOC and RGD gene names that usually represent minor components of the identified clusters were removed, because such entries could not be mapped based on gene names between species. A core set of 32 proteins from our TDP-43 interactome were found in four (DDX3X, DHX9, ELAVL1, HNRNPA0, HNRNPD, HNRNPH1, HNRNPL, HNRNPM, HNRNPR, ILF2, MATR3, PABPC4, RALY, YBX1) or three (DDX5, DDX6, EFTUD2, EIF3A, HNRNPA3, HNRNPAB, HNRNPC, HNRNPK, HNRNPU, HNRNPUL2, ILF3, NONO, NOP56, NOP58, PABPC1, SNRNP200, SNRPA1, SSB) interactome datasets (Blokhuis et al, 2016; Feneberg et al, 2020; Freibaum et al, 2010; Ling et al, 2010). There was also substantial overlap with a BirA proximity labeling dataset for TDP-43 and TDP-25 (Chou et al, 2018), the stress granule proteome (Jain et al, 2016) and TDP-43 myogranules in regenerating muscle (Vogler et al, 2018).

B: Primary rat hippocampal neurons were co-transduced with lentivirus encoding for GFP-tagged TDP-25 variants or GFP and PSM B2-tagRFP lentivirus on day 5. Immunofluorescence images were taken 8 days after transduction (DIV5+8). Counterstain to label nuclei (DAPI). Scale bar $=10 \mu \mathrm{M}$. 


\section{M ovie S1. Mapping M acromolecules to Poly-GA Inclusions, Related to Figure 2}

Tomographic volume and 3D rendering of the neuronal GFP-TDP-25 inclusion depicted in Fig 1C/D. GFP-TDP-25 (red), 26S proteasomes (violet), ribosomes (yellow), TRiC/CCT chaperonins (green), mitochondria in white, ER in pink and other vesicles in light yellow. The macromolecules are mapped to their original positions and orientations, computationally determined by template matching and subtomogram averaging. Proteasomes are enriched within the GFP-TDP-25 inclusions, while ribosomes are mostly found in its periphery. 